\title{
INVESTIGACIONES
}

\section{Comprendiendo el encuentro entre significados del Aprendizaje y Servicio crítico en contextos comparados}

\author{
Understanding the encounter between meanings \\ of Critical Service -Learning in comparative contexts
}

\author{
Ana Zarzuela ${ }^{a}$, Mayka García \\ ${ }^{a}$ Grupo de Investigación HUM230 Eduardo Benot, Universidad de Cádiz. \\ ana.zarzuela@uca.es \\ ${ }^{b}$ Departamento de Didáctica, Universidad de Cádiz. \\ mayka.garcia@uca.es
}

\section{RESUMEN}

Este trabajo compara la experiencia argentina y la española en la articulación del Aprendizaje y Servicio crítico. La investigación desarrollada pretende comprender los significados atribuidos actualmente a estas prácticas en uno y otro lugar, poniendo palabras al recorrido compartido por prácticos e investigadores del Aprendizaje y Servicio en ambos contextos desde una orientación politizada de este tipo de propuestas. Consecuentemente, a través de una metodología cualitativa, interpretativa y etnográfica, se realiza una primera aproximación a la construcción de una historia compartida, a través de un análisis documental. En un segundo momento, utilizando entrevistas y grupos de discusión que hacen emerger las voces de participantes, se consiguen identificar puntos comunes en el desarrollo de este tipo de propuestas. Finalmente se apuntan las claves de un proceso de enriquecimiento mutuo que hacen que el Aprendizaje y Servicio hoy sea una propuesta no solo muy vigente, sino también en expansión.

Palabras claves: Aprendizaje y Servicio, estudio comparativo, aprendizaje mutuo.

\begin{abstract}
This work compares the Argentine and Spanish experience in the articulation of critical Service-Learning. The research has been developed to understand the meanings attributed to these practices in one place and another, putting words to the shared way by practitioners and investigators of Service-Learning in both contexts from a politicized orientation of these types of proposal. Consequently, through a qualitative, interpretive and ethnographic methodology, a first approximation is made to the construction of a shared history, through a documentary analysis. In the second moment, interviews and discussions groups are used to make the voices of participants emerge; common points in the development of this type of proposal are identified. Finally, we present the keys to a mutual enrichment process that makes that today Service-Learning a proposal not only very effective, but also in expansion.
\end{abstract}

Key words: Service-Learning, comparative study, mutual learning. 


\section{INTRODUCCIÓN}

El Aprendizaje y Servicio (ApS) es considerado, hoy en día, un foco de interés pertinente y relevante en la investigación educativa (García \& Cotrina, 2015). En este contexto hemos de precisar que no se trata de una nueva metodología (Batlle, 2013) y que la investigación en Aprendizaje y Servicio tampoco lo es. De hecho pueden identificarse numerosos referentes previos que toman como objeto de indagación el Aprendizaje y Servicio y que se orientan en todas las etapas y niveles del sistema educativo con focos investigativos diversos, como muestran los trabajos de Bringle \& Hatcher (1996), Furco \& Billing (2002), Tapia (2002, 2008), Martínez (2008), Puig (et al. 2007, 2009), Martín, Rubio, Batlle \& Puig (2010), Navarrete, Barrera \& Martín (2010), Ochoa (2010), Eyler \& Giles (1999), Stoecker (2016) o Cate \& Russ-Etf (2017).

Sin embargo, tomar el Aprendizaje y Servicio como objeto de investigación en sí mismo asume relevancia porque esta metodología propia del siglo XXI (Carbonell, 2015) nos permite vislumbrar propuestas educativas en el escenario de los nuevos entornos de aprendizaje en relación (García, 2016), que precisan ser considerados en el marco de los nuevos retos educativos. Entre las bases en las que se sustenta la lógica del avance en el pensamiento en relación con estos nuevos entornos se encuentran ideas tales como: (a) El reconocimiento de que la institución escolar no es el único espacio de educación y, de hecho, como se sostiene a través de la educación expandida (Díaz \& Freire, 2012) actualmente el proceso educativo se articula en procesos menos formalizados; (b) La asunción de la idea de que la institución escolar no es más que un nodo en ese entramado donde se configura la educación y que la escuela necesita conectar con el medio (la comunidad) donde se ancla y compartir un proyecto común (Mayor-Paredes, 2018); y (c) Que dicho proyecto común en relación, implica la comprensión de significados, lo que supone el reconocimiento del otro o de la otra en la legítima convivencia (Maturana, 1992).

La investigación en Aprendizaje y Servicio, en claro auge, puede resultar pertinente, dado que como propuesta metodológica está siendo concebida como una propuesta innovadora (Billig \& Waterman, 2014). Sin embargo, ello la sitúa en el punto de mira para una rápida expansión acrítica que puede vaciarla de sentido, de su esencia (García \& Cotrina, 2015; Robinson, 2000). Ya empiezan a ser cada vez más frecuentes los trabajos que se esfuerzan en diferenciar distintas tendencias dentro de una misma práctica y que nos lleva a enfatizar la distancia entre lo que se denomina "aprendizaje y servicio tradicional" y "aprendizaje y servicio crítico" (Butin, 2015; Deeley, 2016; Latta, Kruger, Payne, Weaver \& VanSickle, 2018; Mitchel, 2008; Terrance, Watkins \& Jimerson, 2018). La investigación es un medio para combatir esta tendencia y es en este contexto de intersección entre relevancia y pertinencia donde cobra sentido la investigación que aquí se presenta, una investigación orientada a indagar en esa esencia del Aprendizaje y Servicio crítico.

Este proceso se realiza a través de un viaje de ida y vuelta entre dos contextos, el español y el argentino. Y se orienta a comprender en profundidad el significado del Aprendizaje y Servicio crítico en ambas geografías, su devenir, las influencias entre ambos países, los aprendizajes compartidos y las proyecciones actuales. Esto, además, se justifica desde la naturaleza de la indagación en Aprendizaje y Servicio en ambos contextos. Y es que, tradicionalmente vemos como las investigaciones europeas sobre el Aprendizaje y Servicio antes referidas, giran alrededor de la sistematización de su utilidad educativa para ponerla en acción en diferentes contextos. Por su parte, las investigaciones latinoamericanas tienden más 
a compartir y difundir experiencias realizadas en diversos espacios, más que a sistematizar resultados. De las potencialidades de la investigación desde estas dos lógicas surge la necesidad de esta, entendiendo la investigación educativa como una herramienta de aprendizaje mutuo y compartido, lo que nos aproxima a un enfoque comunitario (Sandín, 2003).

\section{EL ESCENARIO GENERAL: UNA APROXIMACIÓN AL APRENDIZAJE Y SERVICIO}

La práctica de la conceptualización del Aprendizaje y Servicio se encuentra muy extendida en la literatura científica, donde se presentan compendios definitorios, comparativas y análisis, entre las que destacamos las obras casi fundadoras de Sigmon (1979) que presentaba los componentes básicos del mismo, Furco (1996) que delimitaba el significado de Aprendizaje y Servicio, o Butin (2003) que sistematizaba distintas formas de entender el Aprendizaje y Servicio desde diversas opciones como la técnica, cultural, política y post-estructuralista. Cronológicamente más recientes, pero con un fuerte impacto, nos encontramos los trabajos en este sentido de De la Cerda, Martín, Rovira \& García (2009), que comprendían las referencias clásicas del Aprendizaje y Servicio, añadiendo las aportaciones propias del territorio latinoamericano (especialmente Argentina y Chile) y español; y la de Francisco \& Moliner (2010), que en un sencillo artículo sitúan excepcionalmente el tema.

Se ha estimado que situar el discurso propio, requería avanzar un trabajo de aproximación definitorio al marco conceptual del Aprendizaje y Servicio. Dada la naturaleza de este trabajo, para esto se utilizan como referentes los trabajos de Tapia (2000), Furco \& Billing (2002), Puig et al. (2007) y la Fundación Zerbikas (2008), siendo esta última una propuesta integradora a partir de Rubio en Puig et al. (2007). Estos referentes han sido seleccionados por el impacto académico de sus obras, así como porque representan versiones y voces diversas que significan al Aprendizaje y Servicio en los dos contextos geográficos estudiados, el argentino y el español. También han sido seleccionados porque ofrecen constructos que han sido gestados en contexto educativos de naturaleza diversa, formales y no formales, lo que nos pone en conexión con los supuestos expresados en el anterior introductorio.

Tabla 1. Selección de definiciones relevantes de Aprendizaje y Servicio

\begin{tabular}{|l|l|}
\hline AUTORÍA & DEFINICIÓN \\
\hline Tapia (2000) & $\begin{array}{l}\text { (..) un proyecto educativo solidario protagonizado activamente por lo estu- } \\
\text { diantes, articulado intencionadamente con los contenidos de aprendizaje. (p. 8) }\end{array}$ \\
\hline $\begin{array}{l}\text { Furco \& Billing } \\
(2002)\end{array}$ & $\begin{array}{l}\text { Busca involucrar a los estudiantes en procesos que combinan el servicio a la } \\
\text { comunidad y el aprendizaje académico. (p. 25) }\end{array}$ \\
\hline Puig et al. (2007) & $\begin{array}{l}\text { El ApS es una propuesta educativa que combina procesos de aprendizajes y de } \\
\text { servicio a la comunidad en un único proyecto bien articulado en el que los } \\
\text { participantes aprenden a la vez que trabajan en las necesidades reales del } \\
\text { entorno con la finalidad de mejorarlo. (p. 20) }\end{array}$ \\
\hline
\end{tabular}


Fundación

Zerbikas (2008)
Aprendizaje y Servicio Solidario (AySS) es una metodología que integra el servicio a la comunidad con el aprendizaje de contenidos, habilidades y valores. El AySS funde intencionalidad pedagógica e intencionalidad solidaria. (p.7)

Fuente. Elaboración propia.

Sigmon (1979) nos proporcionaba tres claves definitorias para situar el Aprendizaje y Servicio en el contexto educativo: la participación de los estudiantes, la identificación del servicio como vivido o facilitado y la experiencia de aprendizaje auténtico. En el proceso de análisis de las distintas aproximaciones al concepto y a la práctica de Aprendizaje y Servicio referenciadas se identifican elementos o señas comunes que se presentan a continuación.

Tabla 2. Elementos clave expresados en las distintas conceptualizaciones de Aprendizaje y Servicio tomados como referentes

\begin{tabular}{|l|c|c|c|c|c|c|c|}
\hline & Aprendizaje & Servicio & Finalidad & Necesidad & $\begin{array}{c}\text { Intención } \\
\text { pedagógica }\end{array}$ & Solidaridad & $\begin{array}{c}\text { Papel del } \\
\text { alumnado }\end{array}$ \\
\hline Tapia (2000) & $\bullet$ & & & & $\bullet$ & $\bullet$ & \\
\hline Furco (2002) & $\bullet$ & $\bullet$ & & & $\bullet$ & & $\bullet$ \\
\hline Puig (2007) & $\bullet$ & $\bullet$ & $\bullet$ & $\bullet$ & $\bullet$ & & \\
\hline $\begin{array}{l}\text { Fundación } \\
\text { Zerbikas } \\
\text { (2008) }\end{array}$ & $\bullet$ & $\bullet$ & & & & & \\
\hline
\end{tabular}

Fuente. Elaboración propia.

En esta tabla podemos ver reflejado cómo cada definición presenta rasgos propios. Se quiere hacer notar que en aquellas que nacen en espacios asociativos o de educación social (Puig et al., 2007 y Zerbikas, 2008) tienen menos presente el papel del alumnado (y más el de ciudadano), frente a aquellas que nacen en espacios educativos formales (Tapia, 2000; Furco \& Billing, 2002). Obsérvese cómo todas tienen muy presentes como ingrediente fundamental del ApS los aprendizajes y la intención pedagógica de la metodología. Por su parte, es interesante apuntar que, aunque el servicio es un elemento básico del ApS, no aparece como tal en todas y, este aparece vinculado a veces a una dimensión solidaria.

Podemos apuntar, por otro lado, que encontramos diferentes nomenclaturas para referirse a Aprendizaje y Servicio, cuya génesis formal Barrios, Rubio, Núñez y Vería (2012) sitúan en torno a 1903 en Estados Unidos, de la mano del movimiento de educación cooperativa. A través del análisis de la literatura se observa que en España se designa Aprendizaje y Servicio, Aprendizaje-Servicio y Aprendizaje Servicio; y se nombra en acrónimo como ApS. Por su parte, en Latinoamérica se habla de Aprendizaje y Servicio Solidario, simplificándose como ApSS en Argentina o A+S en Chile. En su versión angloparlante se nombra como Service-Learning o S-L. 


\section{1. ¿DE QUÉ HABLAMOS CUANDO LO HACEMOS DE APRENDIZAJE Y SERVICIO CRÍTICO?}

Teniendo en cuenta lo expresado, asumimos conceptualmente el Aprendizaje y Servicio como una metodología, pedagogía y filosofía de vida (Puig, 2009; Tapia, 2008) que combina los procesos de aprendizaje y el servicio a la comunidad tendiendo lazos entre ambos elementos a través de la reflexión continua durante el proceso. De esta manera no se pierde de vista el verdadero objetivo del ApS: Dar respuesta a necesidades sociales cuestionando la realidad, mejorando los contextos y transformándolos desde la participación activa, cooperativa y horizontal de todas las personas implicadas (alumnado, personas beneficiarias y alianzas), partiendo, al mismo tiempo, desde la solidaridad para construir mejores sociedades y desde la intención pedagógica crítica del propio proceso. Desde esta perspectiva podemos hablar del Aprendizaje y Servicio como una pedagogía revolucionaria (Porfilio \& Hickman, 2011).

Figura 1. Conceptualización gráfica del Aprendizaje y Servicio Crítico

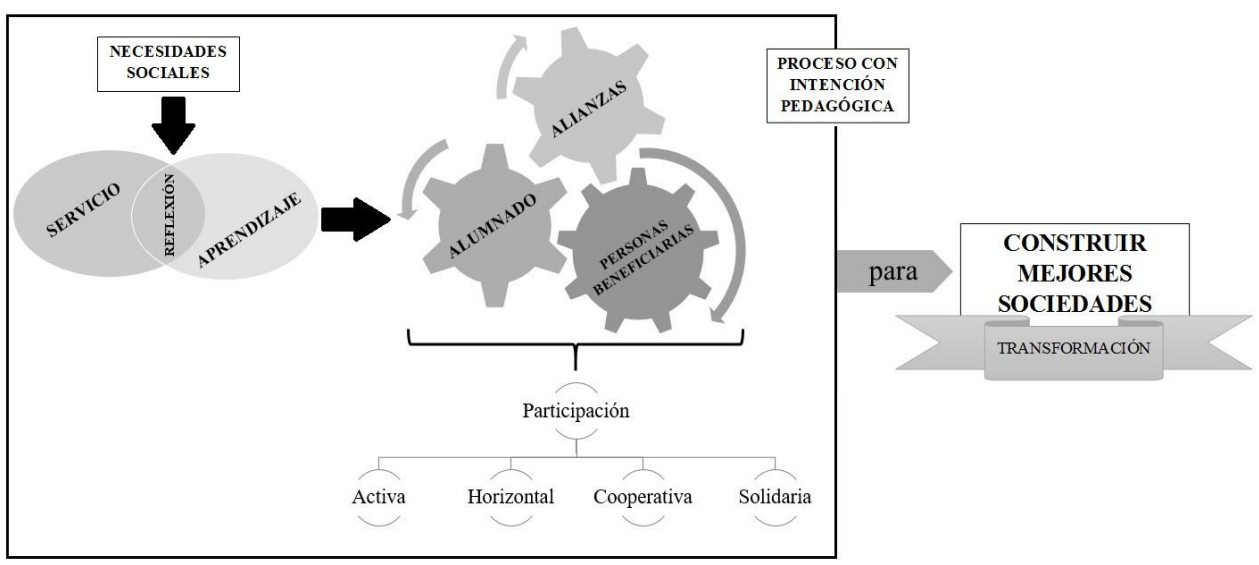

Fuente. Elaboración propia.

Los referentes actuales apuntan que, aunque estemos utilizando diferentes nomenclaturas se identifica una esencia común y es la orientación a transformar la realidad desde la intervención educativa en contextos próximos (García \& Cotrina, 2016; Martínez, 2008; Puig, 2009; Tapia, 2000). Y en esta esencia asume rasgos propios de orientaciones pedagógicas diversas, como la ya clásica hacia la emancipación (Freire, 1970), la valoración de mirada y la voz de niñas y niños (Fielding, 2011; Tonucci, 1997) o el desarrollo de una ciudadanía crítica comprometida (Francisco \& Moliner, 2010). Aquí, bajo el paraguas de una participación auténtica, es donde el Aprendizaje y Servicio crítico, para quienes suscriben este texto, adopta su significado. En este sentido se apuesta por concretar, siguiendo a Mitchell (2008), que, además, el ApS posee una clara orientación inclusiva que nos re-sitúa en la educación emancipadora. De hecho, se asume que la meta última del Aprendizaje y Servicio es "deconstruir los sistemas de poder dando respuesta 
a necesidades a través de un servicio que desmonta las desigualdades que los crean y sostienen" (Mitchell, 2008, p. 50).

En esta línea se encuentran los trabajos Hart (2006) que nos habla del Aprendizaje y Servicio como práctica de libertad y liberación de los silenciados y marginados. Las referencias al Aprendizaje y Servicio Crítico llaman la atención sobre las bases pedagógicas de este tipo de propuestas (Daigre, 2000; Deans, 1999; Giles, 1987; Saltmarsh, 1996) situadas en las aportaciones de Freire y de Dewey. Del primero, a través del Aprendizaje y Servicio se rescata la perspectiva comunitaria y la visión humanista de la educación, que nos habla de voces, de concientización, de empoderamiento y de hecho nomina a este tipo de prácticas con frecuencia como Comunity Service-Learning (Deans, 1999). Del segundo, el Aprendizaje y Servicio Crítico toma la pedagogía experiencial a partir de la cual la acción de aprendizaje (como actividad vivida y pensada) toma sentido, a través de los principios de continuidad e interacción, que ofrecen sentido a la artificialidad de la separación entre teoría y práctica; y de la misma forma sumerge en un ciclo interactivo al aprendizaje y al servicio. Mitchell (2008), además, nos pone en contexto al Aprendizaje y Servicio crítico, porque lo sitúa en un triple escenario interactivo que impregnaría a este tipo de propuestas: su orientación hacia el cambio, la redistribución de poderes y el desarrollo de relaciones auténticas entre las personas participantes.

La revisión de lo anterior nos lleva a conceptualizar que el Aprendizaje y Servicio crítico se construye a partir de 5 pilares fundamentales:

- Una necesidad (social), entendida como situación a la que queremos dar respuesta para mejorarla. Vemos aquí la orientación hacia el cambio, pero también la proactividad de las personas participantes. En este sentido se hace necesario precisar que, como señala Martín (2015) “(...) nos referimos a situaciones de carencia, injusticia o dificultad detectadas en la realidad y que invitan a comprometerse en la mejora de la calidad de vida tanto de personas como de la comunidad o del entorno" (p. 31). A raíz de ello se apunta la inminente necesidad educativa de potenciar una mirada crítica ante las situaciones que nos rodean, lo que nos permita detectar las necesidades y vivir en la sociedad con intencionalidad transformadora a través de la toma de conciencia de las mismas.

- Un servicio, como la acción social que se desarrolla para tratar de ofrecer una respuesta crítica a la necesidad previamente detectada. Ese sentido crítico, tiene que estar alejado de la caridad y centrado y orientado desde la solidaridad que emerge de la toma de conciencia de la injusticia (King, 2004). En este sentido, Puig et al. (2007) matizan que "se requiere que el proyecto ocasione un impacto real" (p. 135). Ello permite evaluar constructivamente y visibilizar la acción en el territorio, lo que supone cuestionar de qué forma se contribuye a la transformación y/o al sostenimiento del status quo.

- Los aprendizajes, como proyecto educativo al servicio de la justicia social (GarcíaPérez \& Gezuraga, 2018). Una propuesta de este tipo debería potenciar la construcción y reconstrucción de aprendizaje significativo y relevante a través de la acción práctica y reflexionada, a la vez que la toma de conciencia de la inequidad, ya que como nos dice Martín (2015) “(...) resulta imposible imaginar una contribución en el entorno que no desencadene experiencias nuevas que modifiquen, amplíen o cuestionen los valores y conocimientos previos" (p. 39). 
- Un partenariado, entendido como alianzas y redes de apoyo que nos ayudarán en menor o mayor medida al diseño, desarrollo y evaluación de los proyectos, que nos sumergen en una perspectiva comunitaria del hecho educativo y en la cultura de la colaboración (Rice \& Pollack, 2000). Y que se genera a través de las prácticas cooperativas que se encuentran en la esencia del Aprendizaje y Servicio y del pensamiento situado en redes de colaboración.

- La reflexión, que supone ser conscientes de qué estamos haciendo en cada momento y por qué lo estamos haciendo de esa manera (Rosenberg, 2000). La reflexión es el punto de encuentro entre aprendizaje y servicio, enriquecido de la acción. De la Cerda et al. (2009) nos devuelven la reflexión como un revivir que aumenta el aprendizaje, lo enriquece. Así mismo es la herramienta que previene la perversión de los proyectos ya sea de la mano del academicismo o del activismo.

Es en este escenario, donde como se puede apreciar, la ética crítica impregna la práctica pedagógica, desde el que se aborda esta investigación que, al igual que la esencia del Aprendizaje y Servicio, reconoce en el otro una oportunidad de aprendizaje y ayuda mutua. De ahí el interés en reconocernos en significados que geográficamente están tan alejados pero que, en esencia, comparten claves.

\section{MÉTODO}

El propósito de esta investigación se concretó en comprender en profundidad cómo ha sido el desarrollo del Aprendizaje y Servicio Crítico en dos contextos diferenciados para identificar las esencias de una construcción conjunta. Por eso, este trabajo se centra en esa conexión aproximando la realidad del Aprendizaje y Servicio en Argentina y España. Este propósito necesitaba dos objetivos investigativos. De un lado, el de construir la historia del Aprendizaje y Servicio en ambos contextos. Y de otro, el de visibilizar aprendizajes mutuos a través de indagación en la influencia del ApS argentino en el español y viceversa. En la tabla posterior se ilustran la relación entre objetivos específicos y cuestiones de investigación que se derivan de lo anterior, pudiéndose observar que dicha relación no es lineal, sino cíclica e interactiva, y que varias cuestiones pueden estar relacionadas con diferentes objetivos.

Tabla 3. Relación entre objetivos y cuestiones de investigación

\begin{tabular}{|l|l|}
\hline Objetivos específicos & \multicolumn{1}{c|}{ Cuestiones de investigación } \\
\hline $\begin{array}{l}\text { A. Comprender el } \\
\text { desarrollo del Aprendizaje } \\
\text { y Servicio crítico }\end{array}$ & $\bullet \quad \begin{array}{l}\text { ¿Cómo se desarrolla el Aprendizaje y Servicio crítico? } \\
\text { ¿Cómo son los contextos en los que se desarrolla la metodología? } \\
\text { ¿Qué condiciones propician el nacimiento del Aprendizaje y } \\
\text { Servicio crítico? }\end{array}$ \\
\hline $\begin{array}{l}\text { B. Identificar sus señas de } \\
\text { identidad }\end{array}$ & $\bullet \quad \begin{array}{l}\text { ¿Qué caracteriza al Aprendizaje y Servicio crítico? } \\
\text { ¿Qué aspectos lo hacen único y propio de cada territorio? }\end{array}$ \\
\hline
\end{tabular}




\begin{tabular}{|l|ll|}
\hline $\begin{array}{l}\text { C. Comparar las } \\
\text { características del } \\
\text { Aprendizaje y Servicio } \\
\text { crítico en ambos espacios }\end{array}$ & $\bullet \quad \begin{array}{l}\text { ¿Qué caracteriza al Aprendizaje y Servicio crítico? } \\
\text { ¿Quectos lo hacen único y propio de cada territorio? }\end{array}$ \\
\hline $\begin{array}{l}\text { D. Establecer rasgos } \\
\text { identificatorios del } \\
\text { Aprendizaje y Servicio de } \\
\text { cada contexto }\end{array}$ & $\bullet \quad$ ¿Qué aspectos lo hacen único y propio de cada territorio? \\
\hline
\end{tabular}

Fuente. Elaboración propia.

El diseño metodológico que orientó la investigación fue cualitativo (Cardona, 2002) pues era necesario comprender, profundizar, interpretar(nos) y llegar a conclusiones a través de los significados que las personas participantes otorgan y otorgamos a su (nuestra) realidad vivida en relación con el Aprendizaje y Servicio. Desde aquí se observa una clara perspectiva interpretativa de corte etnográfico y fenomenológico ya que se investigan contextos concretos, con realidades concretas, y necesitamos introducirnos en ellas para conocerlas y reflexionarlas desde lo vivido (Rodríguez, Gil \& García, 1999). También cabe anunciar el rasgo comunitario (Sadín, 2003) de esta investigación asumiendo premisas de aprendizaje mutuo que orientaba la investigación y que fue referido en apartados anteriores.

\subsection{PARTICIPANTES}

Se ha contado con informantes de ambos contextos geográficos y, para seleccionarlos, se utilizaron como criterios la disponibilidad a participar en esta investigación, la accesibilidad en términos de tiempos, el grado de implicación y experiencia en Aprendizaje y Servicio y el vínculo con las personas participantes. En el caso de informantes en Argentina se ha contado con personas del Centro Latinoamericano de Aprendizaje y Servicio Solidario (CLAYSS), de la Universidad de Buenos Aires y de un centro de educación secundaria. En España han participado personas de la Universidad de Cádiz, la Universidad de Barcelona, de la Asociación ApS(U)CA, de la Red Española de Aprendizaje-Servicio, de la Fundación Zerbikas y del Centre Promotor d'Aprenentatge Servei. La concreción de informantes se presenta en la tabla posterior.

\subsection{RECOGIDA DE INFORMACIÓN}

La primera fase de la investigación supuso una indagación documental que permitió identificar documentos clave que posibilitaron construir un marco conceptual en relación con el nacimiento, la evolución y la acción del ApS en los dos contextos objeto de estudio. Ello permitió abordar una fase de trabajo de campo, que fue desarrollada a lo largo de tres meses y donde se desarrolló la toma de datos a través de: entrevistas semiestructuradas individuales (11) a profesionales que desempeñan su labor en centros promotores de ApS y a docentes de distintos niveles educativos de ambos países, 1 entrevista grupal a alumnado de secundaria, así como 4 grupos de discusión de docentes y estudiantes siendo 
estos últimos desarrollados en el marco de un encuentro internacional sobre Aprendizaje y Servicio desarrollado en Buenos Aires (Argentina).

Tabla 4. Relación de informantes, perfiles y técnicas de recogida de información

\begin{tabular}{|c|c|c|c|}
\hline Informantes Perfil de referencia & Contexto & Técnica & Referencia de codificación \\
\hline $\begin{array}{l}\text { Profesional en Centro } \\
\text { Latinoamericano de Aprendizaje } \\
\text { y Servicio Solidario }\end{array}$ & Argentina & 3 entrevistas & PA 1 \\
\hline $\begin{array}{l}\text { Profesional en Centro } \\
\text { Latinoamericano de Aprendizaje } \\
\text { y Servicio Solidario }\end{array}$ & Argentina & 1 entrevista & PA 2 \\
\hline Docente de Universidad & Argentina & 1 entrevista & PA 3 \\
\hline $\begin{array}{l}\text { Docentes (4) de Centro de } \\
\text { Educación Secundaria }\end{array}$ & Argentina & Grupo de discusión & PA 4 \\
\hline $\begin{array}{l}\text { Estudiantes (6) de Centro de } \\
\text { Educación Secundaria }\end{array}$ & Argentina & 1 entrevista grupal & PA 5 \\
\hline Docentes (4) de Universidad & España & Grupo de discusión & PA6 \\
\hline Docente de Universidad & España & 4 entrevistas & PA 7 \\
\hline Docentes (2) de Universidad & España & 1 entrevista & PA 8 \\
\hline Docentes (3) de Universidad & España & Grupo de discusión & PA 9 \\
\hline $\begin{array}{l}\text { Profesional del Centre Promotor } \\
\text { d'Aprenentarge Servei }\end{array}$ & España & 1 entrevista & PA 10 \\
\hline Estudiantes (3) universitarios & España & Grupo de discusión & PA 11 \\
\hline
\end{tabular}

Fuente. Elaboración propia.

\subsection{ANÁLISIS DE DATOS}

Las entrevistas y los grupos de discusión han sido transcritos y tratados a través de procedimientos informáticos a fin de reducir la información. El análisis de los datos se llevó a cabo a través de un proceso de categorización emergente para así poder reflexionar y comprender en profundidad sin esquemas prefijados. Es importante señalar que, en ocasiones, este procedimiento de análisis no se ha dado de forma lineal, es decir, que la reducción de datos y la interpretación de los mismos han ido de la mano como nos indica Gibbs (2012). 
Para superar los posibles sesgos hemos hecho uso de fuentes de información diversas, invitando a la participación a sujetos de ambos contextos, de etapas y entornos profesionales diferenciados. Contamos con participantes del ámbito asociativo, escolar y universitario, lo que nos ayudaría a cubrir un amplio espectro de prácticas de Aprendizaje y Servicio. Esto se ha considerado en el análisis comparativo de respuestas.

\section{RESULTADOS}

En este momento se plasman los resultados construidos a partir de las voces de las personas participantes. Así, en apartados sucesivos encontraremos una visión comparada de la historia del Aprendizaje y Servicio como metodología, la influencia intercontinental que cruza el océano y la evolución que siguen ambos casos haciendo que esos puentes en común se diluyan, en algunos aspectos, así como la consolidación de la metodología con señas de identidad propias de cada país.

\subsection{EL APRENDIZAJE Y SERVICIO EN ARGENTINA: DE SUS INICIOS, EXPANSIÓN Y SIGNIFICADOS}

En el caso de Argentina comprobamos cómo el Aprendizaje y Servicio es una realidad palpable desde el punto de vista institucional desde 1997 cuando, el propio gobierno federal a través de las políticas educativas del Ministerio de Educación, propone el desarrollo de prácticas de intervención comunitaria como parte del currículum de los centros educativos. Esto sitúa a este país en la vanguardia de la incorporación de este tipo de prácticas. De hecho, es en la década de los 90 cuando empieza a proliferar la sistematización de este tipo de propuestas a través de la investigación en el contexto norteamericano, así como a desarrollarse los primeros centros promotores de Aprendizaje y Servicio (Folgueiras, Luna \& Puig, 2013). Pero cabe destacar que inicialmente el impulso al Aprendizaje y Servicio en el país no vine por vías gubernamentales, sino más bien de la mano de la sociedad civil:

(...) A partir de la reconquista de la democracia en 1983 y ante las recurrentes crisis socioeconómicas (...) un número creciente de instituciones educativas comenzaron a desarrollar proyectos solidarios elaborados pedagógicamente y con mayor impacto social. (...). Prácticamente inventaron el aprendizaje-servicio aún antes de saber que existía una tradición pedagógica que llevaba ese nombre. (PA 2)

La institucionalización y su inserción en los currículo generó una necesidad, la de formación, es por esto que comenzaron a llevarse a cabo diferentes formaciones en torno al Aprendizaje y Servicio con el objetivo de darlo a conocer y difundirlo y, posteriormente, crear redes y compartir experiencias. En esto jugó un papel decisivo el desarrollo del primer Seminario Internacional de Aprendizaje y Servicio Solidario, como foro de encuentro y germen de voluntades conjuntas. Posibilitó visibilizar la metodología en un momento en que cada vez iba va adquiriendo más peso en las instituciones educativas. Tal es así que se crearon varios programas asociados a escuelas fomentados por el propio Ministerio de Educación, entre los que destacan el programa "Escuela y Comunidad", que tenía como uno de sus objetivos particulares "difundir la práctica de la solidaridad como contenido educativo, a través de la metodología del aprendizaje-servicio” (Postay, 2002, p. 191). Hay 
que mencionar que este programa ha sido fuertemente criticado en ocasiones en la medida siendo una propuesta construida arriba-abajo, incentivó el desarrollo acrítico de procesos donde se involucra la solidaridad.

Un hito importante cambio la hoja de ruta, la crisis nacional, que trajo consigo la supresión de gran parte de los programas educativos, lo que afectó de manera notable a este tipo de propuestas, pues tal y como nos relatan “(...) frente a la crisis se produjo una drástica reducción de los programas del Ministerio de Educación” (PA 2). A partir de este momento las instituciones educativas y entidades que desarrollaban procesos de Aprendizaje y Servicio estaban solas. Ya no podían contar con el apoyo federal y debían buscar sus propios recursos para poder continuar caminando. Un soplo de aire fresco llegó en 2002 con la fundación del Centro Latinoamericano de Aprendizaje y Servicio Solidario (CLAYSS), de manos de personas que habían trabajado en el Programa Nacional "Escuela y Comunidad" desde una pedagogía crítica y de la solidaridad (Tapia, 2008). Esto abrió un espacio de referencia y encuentro para aquellas personas que continuaban interesadas en trabajar a través del Aprendizaje y Servicio. Un año más tarde, y con la vuelta a la estabilidad de la presidencia, volvió a establecerse el Aprendizaje y Servicio como política educativa del Ministerio de Educación con la instauración del Programa Nacional "Educación Solidaria":

En 2006, la Ley de Educación Nacional N²6.206 (...) incluyó explícitamente el aprendizaje-servicio y los proyectos educativos solidarios. En 2009, el Consejo Federal de Educación acompañando el proceso de reglamentación de la Ley de Educación Nacional incluyó entre las “Orientaciones para la organización pedagógica e institucional de la educación secundaria obligatoria" las "Propuestas de enseñanza sociocomunitarias", proyectos de aprendizaje-servicio asumidos institucionalmente como cursada obligatoria para al menos un curso (PA 2).

A partir de este momento las instituciones educativas, hasta la Educación Secundaria Obligatoria, no sólo podían, sino que además debían desarrollar propuestas de Aprendizaje y Servicio en sus prácticas pedagógicas. Actualmente y de igual manera que antaño, este proceso continúa apoyado y respaldado por las formaciones desarrolladas en los diferentes Seminarios Internacionales de Aprendizaje y Servicio Solidario que son un referente mundial en el tema:

Se han convertido en uno de los principales foros de actualización, reflexión y formación en la pedagogía del aprendizaje-servicio no sólo en nuestro continente, sino a nivel mundial. Continúan siendo un espacio de encuentro e intercambio horizontal. (PA 3)

Este es el panorama histórico y evolutivo del Aprendizaje y Servicio en Argentina desde sus orígenes, se puede hacer notar que son ya 20 años de impulso, acompañamiento y experiencia, que ha desembocado en un trabajo en red interconectando escuelas y ciudadanía, potenciando la solidaridad y superando el asistencialismo. Quienes participan de este tipo de prácticas quieren destacar que "el aprendizaje-servicio atraviesa las fronteras ideológicas" (PA 2), idea que planteada de esta forma puede ser fuertemente criticada si no se especifica nada más, porque supondría despolitizar la práctica y su poder crítico que hemos referido a través de párrafos precedentes. Por este motivo, cabe ahondar más 
en su significado. Así pues, se ha presentado cómo ha ido creciendo la propuesta, pero aún se ha de desentrañar una clave: ¿cómo es ese Aprendizaje y Servicio? Son muchas las oportunidades ofrecidas por las personas participantes en esta investigación para dar respuesta a esta cuestión. La siguiente voz, se considera que resumen bien el espíritu vivido:

Cómo lo llamemos es lo de menos. Lo importante es que reúna las características básicas para que sea aprendizaje-servicio. (...) que sea solidario porque no sirve cualquier servicio, es fundamental que sea construido CON la sociedad civil y no PARA la sociedad civil para garantizar la construcción conjunta a través del diálogo, la horizontalidad y el trabajo mancomunado. (...) es básico el papel del alumnado en los procesos de aprendizaje-servicio ya que deben ser los protagonistas para apropiarse de la pedagogía y darle sentido. (...) siempre debe haber una planificación intencionada de los aprendizajes. (PA 2)

Se han destacado estas palabras porque permite rescatar esencias, que es precisamente el objetivo del estudio. En ellas encontramos cómo, a través del Aprendizaje y Servicio en el contexto argentino, pone el énfasis en esa importancia de superar el asistencialismo teniendo en cuenta a las personas beneficiarias como agentes activos en la transformación de su realidad. Por otro lado, en ese cambio de roles que precisa la trasformación de la educación más tradicional donde el alumnado es el receptor pasivo del conocimiento. A partir de aquí se da paso al reconocimiento y al trabajo entre iguales desde la horizontalidad. Esto supone la asunción de que el conocimiento se construye en comunidad y en la reciprocidad de las conciencias.

Otro elemento a examen en este devenir argentino del Aprendizaje y Servicio tiene que ver con su institucionalización, cuyos riesgos de vaciado de sentido parecen ser solventados a través de la combinación de obligatoriedad y opcionalidad, que el alumnado asume cuando ha descubierto el poder que tiene como agente de transformación:

Trabajando a través del Aprendizaje y Servicio ayudamos a mejorar diferentes espacios. El primer año es obligatorio y en el segundo año puedes participar como alumna externa que ayuda en la coordinación del proyecto y, como nos gustó tanto, repetimos. Podemos transformar las cosas y camina hacia la justicia social. Si no lo hacemos desde la escuela, ¿desde dónde lo haremos? (PA 5)

Esto posibilita que el alumnado sea constructor de procesos, que le otorguen sentido al acto pedagógico (y al de aprendizaje) y se empoderan en un proceso en el que se les devuelve una imagen positiva y fuerte. Es aquí cuando ese camino hacia la justicia social cobra vida y el ApS traspasa los límites de lo educativo (tradicionalmente hablando). Lo educativo se enriquece, de un lado, de aprendizaje de y en solidaridad, a partir de experiencias vividas y reflexionadas, así como se abren espacios de liderazgo estudiantil que emanan de prácticas de toma de conciencia y acción cooperativa. Se tratada, en esencia, de atribuir a la educación el poder de conjugar experiencias educadoras que posibiliten la emancipación y la transformación social a través de experiencias de servicio social y comunitario. En lo anterior podemos una clara influencia de las bases de Dewey y Freire.

Para finalizar esta puesta en situación cabe hacer notar las continuas alusiones en las entrevistas a que las anteriores son las bases desde las que se construye del Aprendizaje y 
Servicio en escuelas y universidades argentinas. Nos recuerdan la necesidad de empoderarnos a través de la educación, de tomar los contextos para transformarlos, teniendo en cuenta a todas las personas y de caminar hacia la construcción de una sociedad en la que todas las personas tengan cabida creando espacios más justos, democráticos, equitativos y solidarios.

\subsection{SU "TRASFERENCIA" AL CONTEXTO ESPAÑOL: DE SUS ORÍGENES Y DEL POSTERIOR PROCESO DE DESCENTRALIZACIÓN}

El Aprendizaje y Servicio en España comienza a conocerse en profundidad a partir de Batlle (2013), puesto que una persona clave, asume a través de un proceso becado el reto de impulsora este tipo de propuestas en el territorio. Es precisamente la fuente argentina de la que bebe dicha persona, así como otro conjunto de impulsores/as que se sitúan en el contexto universitario.

Sería injusto no señalar, como indican las personas informantes de la investigación que de manera previa existían prácticas de este tipo. Sin embargo, también hay que valorar que fue el hecho de que una persona comenzara a poner nombre al Aprendizaje y Servicio y asumiera la difusión de esta metodología adscrita a la práctica de solidaridad, lo que actuó como desencadenante. De hecho, señala que no tenemos que reconocer en este tipo de propuestas un invento, sino más bien el descubrimiento de una posibilidad. En esta línea algunas de las personas participantes en la investigación nos comentan que:

Cuando el profesorado oye hablar por primera vez de ApS reconoce en su práctica docente o en la de algunos de sus colegas actividades semejantes o como mínimo con objetivos sociales y de implicación en territorios muy parecidos. (PE7 y PE8)

Destacando lo anterior, observamos como la difusión del Aprendizaje y Servicio en España se ha guiado, en todo momento, bajo un principio fundamental, el de realizar una construcción de abajo-arriba a través del contagio, que parte de un reconocimiento y valoración de las propuestas de aprendizaje experiencial, prácticas de campo y de trabajo social, etc. Y es así como se han ido descubriendo prácticas de Aprendizaje y Servicio en diferentes puntos del territorio dándolas a conocer y poniéndolas en valor para favorecer la creación redes de intercambio y poder crear diversos núcleos promotores del Aprendizaje y Servicio en este país. En el caso español, el Aprendizaje y Servicio no se encuentra inserto como propuesta educativa ni como acción social en el currículo oficial. Esto le otorga un carácter descentralizado. Por esto, el proceso de expansión y desarrollo ha venido de la mano de las personas que le han dotado de valor y que han ido configurando grupos territoriales, en primera instancia apoyados por entidades asociativas.

En un segundo momento, las universidades han jugado un papel primordial en la difusión de las prácticas, de su metodología y en la conformación de grupos motores. En 2010, con objeto de conectar a los núcleos territoriales, nace la Red Española de Aprendizaje-Servicio siendo formada por la unión de fuerzas de los grupos promotores territoriales y teniendo identidad de red de redes pues,

(...) ya en este momento inicial formulamos expectativas de relación que iban más allá de la simple coexistencia, como el deseo de compartir herramientas didácticas y expertos internacionales. (PE 10) 
Otro claro ejemplo de la descentralización del Aprendizaje y Servicio en España es que, además de la Red Española de Aprendizaje-Servicio, contamos con la Red Universitaria Española de Aprendizaje-Servicio, también fundada en 2010. Igualmente, podemos destacar que, a día de hoy, la expansión del Aprendizaje y Servicio es tal que contamos con grupos promotores en todas las Comunidades Autónomas de España, como podemos ver en la siguiente figura donde se presenta la expresión gráfica de este dato, situado cronológicamente.

Figura 2. Grupos promotores de ApS en España

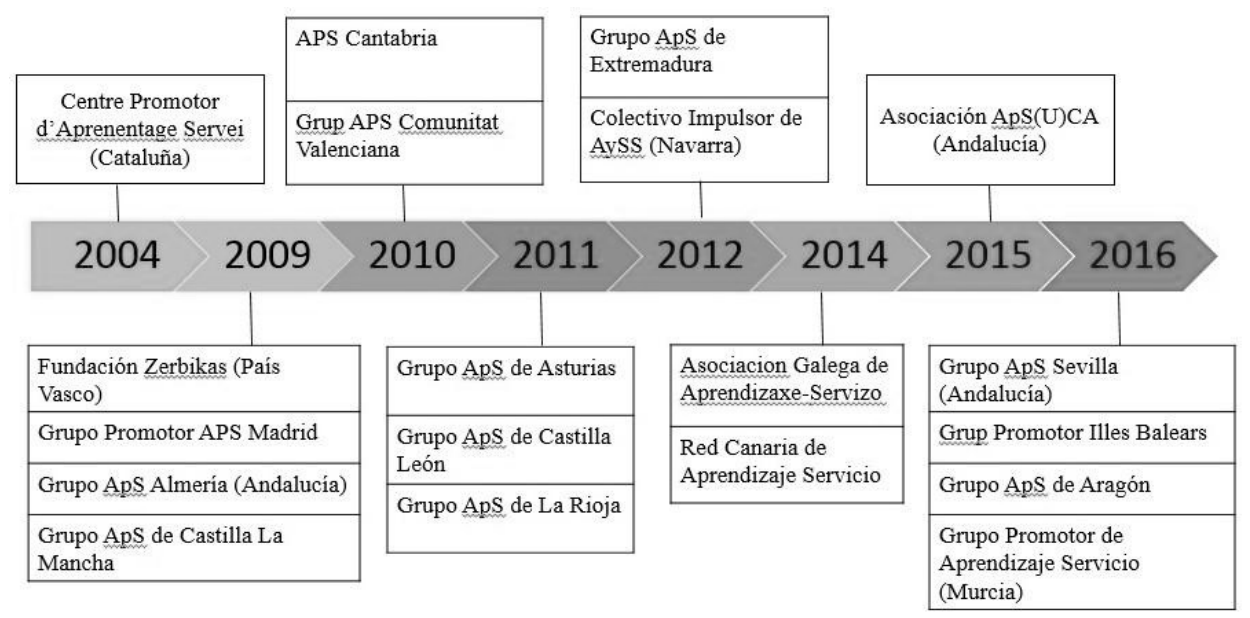

Fuente. Elaboración propia.

En este país, la tradición en Aprendizaje y Servicio es más fuerte en el contexto de las universidades, aunque cada vez son más escuelas las que se unen a este movimiento. Esto es así porque, como destacan las personas participantes, en ellas aún existe una fuerte conciencia crítica, aunque se contradiga con sus propias dinámicas.

(...) cuando la Universidad opta por incorporar estrategias de ApS va a la sociedad para escuchar, para conocer sus necesidades reales y construir desde la docencia y la investigación para mejorarla. (PE7 y PE8)

En esta dirección, nos han formulado aportaciones que nos invitan a pensar que el Aprendizaje y Servicio, en muchas ocasiones, traspasa los límites de ser una metodología y llega a convertirse en una filosofía de vida que permite apoyar el desarrollo personal a través de propuestas pedagógicas de quienes aspiran a contribuir a la construcción de comunidades democráticas, justas y equitativas:

(...) aproximarse al Aprendizaje y Servicio supone un descubrimiento apasionado de estar, el hacer y el ser con sentido público en educación: un sentido cívico, cooperativo, inclusivo, solidario, transformador y crítico. (PE 6) 
(...) resuelve problemas inmediatos centrando su mirada en el contexto para que este sea estimulante y proyecte a ciudadanos críticos, tolerantes, solidarios que busquen la libertad en base al amor y la felicidad de todos. (PE 11)

Y es habilitar procesos y contextos que posibiliten educar la mirada con la que observamos andamiándolos de una perspectiva crítica, sienta las bases de la toma de conciencia de la opresión, del individualismo atroz en el que vivimos, entre otros asuntos clave. Poner en práctica el Aprendizaje y Servicio nos permite construir comunidades desde la cooperación y no desde la competición. De esta manera todas las personas son importantes para el desarrollo comunitario.

Un buen servicio requiere reconocer al otro como persona con dignidad, no como destinatario pasivo de aquello que queremos entregarle. Se trata de una persona o grupo de personas que tiene voz y opinión acerca de lo que se les ofrece. (...) El reconocimiento se abre a la reciprocidad: quien recibe también es capaz de dar. (PE 10)

Esta manera de construir comunidad cualitativamente distinta a las prácticas cotidianas nos permite hacerlo desde la horizontalidad, donde todas las voces son escuchadas y tenidas en cuenta, poniendo así en valor a todas las personas que participan de esa construcción conjunta.

(...) en un mismo planteamiento pedagógico confluyen la determinación metodológica por el aprendizaje activo y el propósito de abrir la institución educativa al medio, el invento del aprendizaje servicio está casi servido. El "casi" es porque falta todavía un ingrediente esencial: en impulso ético y social de contribuir directamente a la mejora de la comunidad. (PE 10)

En esta última expresión se ponen de manifiesto algunos de los elementos claves de la metodología. Ya hemos comentado que en su esencia deben existir aprendizajes ligados al servicio y, para que verdaderamente sea Aprendizaje y Servicio crítico, los propios participantes deben descubrir necesidades sociales, lo que permite tomar conciencia de las mismas e imaginar colectivamente formas de abordarlas desde una ética y práctica solidaria. Y es que “(...) el Aprendizaje y Servicio es experiencia viva a reflexionar y teorizar. Supone pensar en lo que hacemos combinando la reflexión y la acción.” (PE9).

Como vemos, es las personas informantes presentan como fundamental la necesidad de un equilibrio entre los elementos del Aprendizaje y Servicio, de tal forma que no caigamos en el academicismo o en el activismo balanceando el proceso. Pero es en el papel que juega la reflexión el que ha emergido clave entre quienes apuestan por esta metodología desde una opción crítica, un papel que debe acompañar la propuesta en su totalidad, lo que como señalan Páez y Puig (2013) implica tratar de maximizar los formatos de acción para hacerlo posible.

\subsection{SIGNIFICADOS COMPARTIDOS: LA PEDAGOGÍA CRÍTICA COMO ESENCIA}

Analizando el desarrollo del Aprendizaje y Servicio en los territorios estudiados, emerge como rasgo común la vivencia de que estemos hablando no solo de una metodología, sino una filosofía de vida en sí misma. En este sentido, las personas que lo promueven 
lo hacen desde el convencimiento y la pasión de pensar que es una forma de contribuir al desarrollo de una ciudadanía solidaria a través de las escuelas. Lo hacen también dotando los procesos de espacios para la reflexión continua y resalta la importancia otorgada en ello a la horizontalidad, que se refiere a una relación de participación igualitaria, desde el punto de vista de los poderes.

En ambos escenarios han existido referencias a la justicia social, a aprender haciendo, a ciudadanía activa, a compromiso, a inclusión socioeducativa, etc. Y en su desarrollo se reconocen influencias múltiples que sustentan su esencia diversa. En común tienen esas esencias pedagógicas la orientación crítica.

(...) no evoluciona a partir de una única corriente pedagógica, sino que avanza desde trayectorias e intereses diversos. (...) Es aquí en el caldo de cultivo de estas pedagogías Rousseau, Freire, Dewey, Freinet, ... - donde han podido fructificar las propuestas. (PE 10)

Actualmente, el Aprendizaje y Servicio es una práctica extendida en ambos países, muy extendida de hecho, y ambas geografías comparten también que existan fuertes entidades sociales que dan cobertura y promoción a este tipo de prácticas y que son legitimadas desde el punto de vista científico. Un aspecto diferencial, sin embargo, tiene que ver con los intereses de investigación en uno y otro lugar. Hoy en día, la preocupación investigativa en torno al Aprendizaje y Servicio en España gira alrededor de la sistematización e institucionalización del mismo, precisamente porque contextualmente es rasgo diferencial con la experiencia de Argentina.

Otro tópico de interés es el referido al movimiento estudiantil que se está desarrollando en España de la mano del Aprendizaje y Servicio. El alumnado es el centro de la metodología y toma fuerza en los procesos. Sin embargo, esto no es suficiente y es necesario que, poco a poco, el alumnado de diferentes puntos del país también establezca redes de apoyo entre sí y se hagan fuertes pues "detectaron la necesidad de construir conjuntamente espacios de diálogo, reflexión y toma de decisiones sobre ApS" (PE 11). A pesar de que en la literatura y en las propias prácticas de aula es el alumnado quien lidera los procesos de Aprendizaje y Servicio se puede comprobar como en los foros y espacios de toma de decisiones su presencia es muy escasa. Desde que se inició este proceso liderado por el alumnado cada vez son más y más estudiantes quienes asisten a diferentes encuentros, quienes alzan su voz y se posicionan en primera persona con relación al Aprendizaje y Servicio.

\section{DISCUSIÓN Y CONCLUSIONES}

Podemos empezar señalando que en ambos países se desarrollaban experiencias de Aprendizaje y Servicio antes de darle tal nombre (Battle, 2013; Ministerio de Educación de la Nación, 2017). En Argentina esto viene de la mano de esa tradición en voluntariado que existe en Latinoamérica (Tapia, 2002, 2008) mientras que en el caso español se asocia a las inquietudes trabajar con la comunidad a través de la innovación (García \& Cotrina, 2015, 2016; Puig, 2009; Rubio \& Escofet, 2017). Además, se puede hacer notar que, aunque reciban nomenclaturas diferentes en cada país, tienen la misma connotación crítica cuando se asienta en la solidaridad, en reciprocidad de conciencias y se articulan desde la horizontalidad (García \& Cotrina, 2016; Puig, 2009; Puig et al., 2009; Tapia, 2002, 2008). 
Otro aspecto común que viene dado en ambos contextos es que se impulsan propuestas de Aprendizaje y Servicio a partir de situaciones de crisis socioeconómica. En Argentina, nace el Centro Latinoamericano de Aprendizaje y Servicio Solidario en el 2006 (Ministerio de Educación de la Nación, 2017) y, en España, los grupos impulsores, la Red Española de Aprendizaje y Servicio y la Red Universitaria Española de Aprendizaje y Servicio (Batlle, 2013).

En ambas geografías se comparten preocupaciones y, en este sentido, una de ellas sigue siendo el impulsar la formación y la difusión de la metodología. También existe un especial interés en evidenciar los resultados de su implementación, por lo que cada vez son más las investigaciones (que actualmente están centradas en la institucionalización), congresos y publicaciones existentes en este sentido. De esta forma se comparten las experiencias inspiradoras para difundir el Aprendizaje y Servicio a través del contagio y el cambio pedagógico en las instituciones educativas (Batlle, 2013).

Pero también son muchas las diferencias entre la puesta en práctica del Aprendizaje y Servicio a ambos lados del Océano Atlántico. En Argentina las prácticas del Aprendizaje y Servicio Solidario vienen impulsadas y regladas desde el propio Ministerio de Educación (Ministerio de Educación de la Nación, 2017), lo que implica un proceso de arriba-abajo y centralizando la metodología. En el territorio español el Aprendizaje y Servicio se pone en práctica introduciendo la educación social en las escuelas y en las universidades (Batlle, 2013). A diferencia de en el caso anterior, aquí es el propio profesorado el que descubre estas prácticas y decide ponerla en práctica (proceso de abajo-arriba). Así mismo, el Aprendizaje y Servicio se encuentra descentralizado al contar con tantos grupos impulsores y redes, que redistribuyen impulsos, conocimientos y experiencias.

Por otra parte, vemos como en Argentina la mayor tradición en Aprendizaje y Servicio se da en las escuelas (Ministerio de Educación de la Nación, 2017) y las entidades sociales y, poco a poco y gracias al contagio, se ha ido extendiendo su puesta en práctica también a las universidades (Horacio, 2006). Por el contrario, en España la mayor puesta en práctica del Aprendizaje y Servicio viene a manos de las universidades (García \& Cotrina 2015; Martínez, 2008; Rubio \& Escofet, 2017). Sin embargo, las responsables de su extensión a la escuela son sobre todo las entidades sociales, en las que se integran de igual manera académicos.

Un rasgo definitorio de cada espacio, lo encontramos en sus tradiciones de trabajo con la comunidad. Como podemos ver, la tradición cultural de cada contexto influye directamente en la configuración del Aprendizaje y Servicio, haciendo que esas peculiaridades formen parte de sus señas de identidad. Vemos como en Argentina los proyectos están relacionados con situaciones de opresión, injusticia, los pueblos originarios, etc. y a través del apoyo y respaldo del Ministerio de Educación existen becas y los premios que permiten visibilizar y poner en práctica la pedagogía. En el caso de España, podemos comprobar como esa descentralización ha potenciado el establecimiento de redes de apoyo e intercambio de diversos espacios favoreciendo las relaciones entre Comunidades Autónomas. En este sentido vemos como ese intercambio va mucho más allá, pues ya son muchos los ayuntamientos y gobiernos de diferentes municipios se interesan por la metodología y se preocupan por conocer el ApS, desde su vertiente más social, para ponerlo también en práctica y apoyar los procesos que se desarrollan. Con esto se pone en valor el trabajo de todas estas personas que trabajan el Aprendizaje y Servicio y alcanza el fin último del proceso de contagio. 
Como se pone de manifiesto a lo largo de la investigación, el Aprendizaje y Servicio crítico argentino y español no son dos realidades completamente extrañas entre sí, y es que el origen del Aprendizaje y Servicio español viene del otro lado del océano, precisamente de Argentina. Una vez que este desembarca en las prácticas pedagógicas españolas comienzan a transformarse para ajustarse a la nueva realidad a la que debe dar respuesta, por lo que, aunque el punto de partida sea el mismo desemboca en dos devenires diferentes.

Finalmente se quiere añadir que esta devolución de la mirada a Latinoamérica a través de un proceso de investigación de naturaleza comparativa nos permite romper con esa tradición colonial europea, sostenida en imposiciones de conocimiento y de lectura de estos. Se considera que este trabajo contribuye a poner en valor el trabajo realizado en Argentina y nos permite abrirnos a otros escenarios desde el reconocimiento de sus contribuciones. A la vez, nos permite identificar la esencia de las prácticas de Aprendizaje y Servicio que se sostienen desde pilares críticos, a través del reconocimiento del otro y en el otro en el contexto español. Trabajos como este nos dan la oportunidad de "mantener una distancia respecto a la tradición eurocéntrica que equivale a ser consciente del hecho de que la diversidad de la experiencia del mundo es inagotable, y que, por consiguiente, no la puede explicar ninguna teoría general única” (De Sousa Santos, 2017, p. 75).

\section{REFERENCIAS BIBLIOGRÁFICAS}

Barrios, S., Rubio, M., Núñez, M. G., \& Vería, C. S. (2012). Aprendizaje-servicio como metodología para el desarrollo del pensamiento crítico en educación superior. Revista Cubana de Educación Médica Superior, 26(4), 594-603.

Batlle, R. (2013). El Aprendizaje-Servicio en España: El contagio de una revolución pedagógica necesaria. Madrid: PPC.

Billig, S. H., \& Waterman, A. S. (Eds.). (2014). Studying service-learning: Innovations in education research methodology. London: Routledge.

Bringle, R., \& Hatcher, J. (1996). Implementing Service Learning in Higher Education. Journal of Higher Education, 67(2), 221-239.

Butin, D. (2003). Of what use is it? Multiple conceptualizations of service learning within education. Teachers College, 105(9),1674-92.

(2015). Dreaming of justice: Critical service-learning and the need to wake up. Theory Into Practice, 54(1), 5-10.

Carbonell, J. (2015). Pedagogías del siglo XXI: Alternativas para la innovación educativa. Barcelona: Octaedro.

Cardona, C. (2002). Introducción a los Métodos de Investigación en Educación. Madrid: Editorial EOS Universitaria.

Cate, R., \& Russ-Eft, D. (2017). A Review of Current Methods to Develop Empowering ServiceLearning Programs for Latina/o College Students. Journal of Hispanic Higher Education, 17(2). DOI: https://doi.org/10.1177/1538192717729735

Daigre, E. (2000). Toward a critical service-learning pedagogy: A Freirean approach to civic literacy. Academic Exchange Quarterly, 4(4), 6-14.

De la Cerda, M., Martín, M., Martín, X., Puig, J. M. \& García, A. (2009). Aprendizaje servicio: ejemplos y definiciones. En J.M. Puig (Coord.) Aprendizaje servicio (ApS): educación y compromiso cívico (pp. 15-32). Barcelona: Graó.

De Sousa Santos, B. (2017). Más allá de la imaginación política y de la teoría crítica eurocéntricas. Revista Crítica de Ciências Sociais, (114), 75-116. 
Deans, T. (1999). Service-Learning in Two Keys: Paulo Freire's Critical Pedagogy in Relation to John Dewey's Pragmatism. Michigan Journal of community service learning, 6, 15-29.

Deeley, S. J. (2016). El Aprendizaje-Servicio en educación superior: Teoría, práctica y perspectiva crítica. Madrid: Narcea Ediciones.

Díaz, R. \& Freire, J. (2012). Educación expandida. Sevilla: Zemos98.

Eyler, J. \& Giles, D. (1999). Where's de Learning in Service-Learning? San Francisco: Jossey-Bass.

Eyler, J. \& Giles Jr, D. E. (1999). Where's the Learning in Service-Learning? Jossey-Bass Higher and Adult Education Series. Jossey-Bass, Inc., 350 Sansome St., San Francisco, CA 94104.

Fielding, M. (2011). La voz del alumnado y la inclusión educativa: una aproximación democrática radical para el aprendizaje intergeneracional. Revista Interuniversitaria de Formación del Profesorado, 70(25.1), 31-61.

Folgueiras, P., Luna, E., \& Puig, G. (2013). Aprendizaje y servicio: estudio del grado de satisfacción de estudiantes universitarios. Revista de Educación, 362, 159-185.

Francisco, A., \& Moliner, L. (2010). El Aprendizaje Servicio en la Universidad: una estrategia en la formación de ciudadanía crítica. Revista electrónica Interuniversitaria de Formación del Profesorado, 13(4), 69-78.

Freire, P. (1970) La pedagogía del oprimido. Madrid: Siglo XXI.

Fundación Zerbikas (2008). Aprendizaje y Servicio Solidario. Guía de bolsillo. Bilbao: Fundación Zerbikas.

Furco, A. (1996). Service-learning: A balanced approach to experiential education. Recuperado de https://digitalcommons.unomaha.edu/cgi/viewcontent.cgi?article=1104\&context=slceslgen

Furco, A, \& Billing, S. (ed) (2002) Service-Learning. The essence of the Pedagogy. Greenwich CT: Information Age Publishing.

García, R. J. (2016). Nuevos escenarios de aprendizaje en nuevos entornos de relación. El Diario de la Educación, 25-10-2016. Recuperado de http://eldiariodelaeducacion.com/blog/2016/10/25/ nuevos-escenarios-aprendizaje-nuevos-entornos-relacion/

García, M., \& Cotrina, M. (2015). El aprendizaje y servicio en la formación inicial del profesorado: De las prácticas educativas críticas a la institucionalización curricular. Revista de Currículum y Formación del Profesorado, 19(1), 1-6.

(2016). Aprendizaje y servicio: una herramienta pedagógica inclusiva para "vivir" la transformación en el marco de la formación inicial del profesorado. En M. Fernández \& N. Alcaraz (Coord.) Innovación Educativa. Más allá de la ficción (pp. 175-187). Madrid: Pirámide.

García-Pérez, A. \& Gezuraga, M. (2018). ABProblemas, ABProyectos, ABRetos... ¿Legado de Dewey? Valor añadido del ApS respecto a "otros" aprendizajes experienciales. En V. Martínez, N. Melero, E. Ibález \& M.C. Sánchez (Eds.) El Aprendizaje-Servicio en la Universidad. Una metodología docente y de investigación al servicio de la justicia social y el desarrollo sostenible. (pp. 22-27). Salamanca: Comunicación Social.

Gibbs, G. (2012). El análisis de datos cualitativos en Investigación Cualitativa. Madrid: Morata.

Giles, D. E. (1987). Dewey's theory of experience: Implications for service-learning. Journal of Cooperative Education, 27(2), 87.

Hart, S. (2006). Breaking literacy boundaries through critical service-learning: Education for the silenced and marginalized. Mentoring \& Tutoring, 14(1), 17- 32.

Horacio, N. (2006). Aprendizaje y Servicio en Educación Superior. La experiencia latinoamericana. Seminario Internacional de Responsabilidad Social Universitaria. Aprendizaje Servicio. Presentación "Seminario Internacional Responsabilidad Social. Universitaria: Aprendizaje Servicio" - Caracas, Abril 2006.

King, J. T. (2004). Service-learning as a site for critical pedagogy: A case of collaboration, caring, and defamiliarization across borders. Journal of Experiential Education, 26(3), 121-137.

Latta, M., Kruger, T. M., Payne, L., Weaver, L. \& VanSickle, J. L. (2018). Approaching Critical Service-Learning: A Model for Reflection on Positionality and Possibility. Journal of Higher 
Estudios Pedagógicos XLVI, N 1: 57-77, 2020

COMPRENDIENDO EL ENCUENTRO ENTRE SIGNIFICADOS DEL APRENDIZAJE Y SERVICIO CRÍTICO EN CONTEXTOS COMPARADOS

Education Outreach and Engagement, 22(2), 31-55.

Martín, X. (2015). El aprendizaje servicio articula tres dinamismos educativos básicos: necesidades, servicio y aprendizaje. En J.M. Puig (Coord.). 11 ideas claves. ¿Cómo realizar un proyecto de aprendizaje servicio? (pp. 29-76). Barcelona: Graó.

Martín, X., Rubio, L., Batlle, R. \& Puig, J. M. (2010) ¿Qué es el aprendizaje servicio? En Martín, X. y Rubio, L. (Coord). Prácticas de ciudadanía. Diez experiencias de aprendizaje servicio (pp.1524). Madrid: Ediciones Octaedro.

Martínez, M. (2008). Aprendizaje servicio y responsabilidad social de las universidades. Barcelona: Ediciones Octaedro.

Maturana, H. (1992). Emociones y Lenguaje en Educación y Política. Santiago de Chile: Ediciones Pedagógicas.

Mayor-Paredes, D. (2018). Prácticas de aprendizaje-servicio como escenarios de confluencia entre la educación escolar y social. Revista Iberoamericana de Educación, 76, 35-56.

Ministerio de Educación de la Nación (2017). Antología Volumen 1. Actas de los Seminarios Internacionales de Aprendizaje y Servicio Solidario (2010-2016). República Argentina: Ministerio de Educación de la Nación, Unidad de Programas especiales, Programa Nacional Educación Solidaria.

Mitchell, T. D. (2008). Tradicional vs. Critical Service-Learning: Engaging the Literature to Differentiate Two Models. Michigan Journal of Community Service Learning, 50-65.

Navarrete, A., Barrera, M. E. \& Martín, M. (2010). Evaluación de proyectos de servicio social en una universidad mexicana. magis, Revista Internacional de Investigación en Educación, 2(4), 371-382.

Ochoa, E. (2010). Aprendizaje-Servicio en América Latina: Apuntes sobre pasado y presente. Tzhoecoen (Edición especial dedicada al Aprendizaje de Servicio), 5, 108-125.

Páez, M., \& Puig, J. M. (2013). La reflexión en el Aprendizaje-Servicio. Revista internacional de educación para la justicia social, 2(2), 13-32.

Porfilio, B. \& Hickman, H. (2011). Critical Service-Learning as Revolutionary Pedagogy: A Project of Student Agency in Action. Charlotte, NC: Information Age Publishing.

Postay, V. (2002). Las escuelas solidarias del modelo neo-neo y la formación de la cultura política: el caso del Programa Nacional de Escuela y Comunidad. Cuadernos de Educación, Universidad de Córdoba, 189-201.

Puig, J. M. (2009). Aprendizaje Servicio (ApS). Educación y compromiso cívico. Barcelona: Grao.

Puig, J. M., Batlle, R.; Bosch, C. \& Palos, J. (2007): Aprendizaje servicio. Educar para la ciudadanía. Barcelona: Octaedro.

Rice, K. \& Pollack, S. (2000). Developing a critical pedagogy of service learning: Preparing self-reflective, culturally aware, and responsive community participants. En C. R. O'Grady (Ed.), Integrating service learning and multicultural education in colleges and universities (pp. 115-134). Mahwah, NJ, US: Lawrence Erlbaum Associates Publishers.

Robinson, T. (2000). Service learning as justice advocacy: Can political scientists do politics? Political Science and Politics, 33(3), 605-612.

Rodríguez, G., Gil, J. \& García, E. (1999). Metodología de la Investigación cualitativa. Málaga: Ediciones Aljibe.

Rosenberger, C. (2000). Beyond empathy: Developing critical consciousness through service learning. In C. R.O'Grady (Ed.),Integrating service learning and multi-cultural education in colleges and universities (pp. 23-43). Mahwah, NJ: Lawrence Erlbaum Associates.

Rubio, L., \& Escofet, A. (Coord.) (2017) Aprendizaje-Servicio (ApS): Claves para su desarrollo en la Universidad. Barcelona: Octaedro-ICE.

Saltmarsh, J. (1996). Education for critical citizenship: John Dewey's contribution to the pedagogy of community service learning. Michigan Journal of Community Service Learning, 3(1), 13-21.

Sandín, M.P. (2003). Investigación cualitativa en educación. Fundamentos y tradiciones. Madrid: McGraw and Hill. 
Sigmon, R. L. (1979). Service-learning: Three principles. Synergist, 8(1), 9-11.

Stoecker, R. (2016). Liberating Service Learning and the Rest of Higher Education Civic Engagement. Philadelphia, PA: Temple University Press.

Tapia, N. (2000) La solidaridad como pedagogía. Buenos Aires: Ciudad Nueva.

. (2002). El aprendizaje-servicio en América Latina, en Aprender sirve, servir enseña, Buenos Aires: Centro Latinoamericano de Aprendizaje-Servicio Solidario. . (2008). Aprendizaje y servicio solidario. Buenos Aires: Ciudad Nueva.

Terrance, T. C., Watkins, M. L., \& Jimerson, L. (2018). Critical Service-Learning and Cultural Humility: Engaging Students, Engaging Communities. En O. Delano, M. Penick \& S. Frondie (Eds.) Culturally Engaging Service-Learning with Diverse Communities (pp. 1-19). IGI Global.

Tonucci, F. (1997). La verdadera reforma empieza a los tres años. Revista Investigación en la Escuela, 33, 5-16. 
